\title{
BISIMPLE WEAKLY INVERSE SEMIGROUPS WITH PARTIAL RIGHT UNITOIDS
}

\author{
by S. MADHAVAN
}

(Received 11 September, 1984; revised 10 May, 1986)

1. Introduction. In an earlier paper [5] of the author bisimple weakly inverse semigroups with partial identities were studied. The aim of this paper is to extend the results to a wider class of semigroups, viz: bisimple weakly inverse semigroups with partial right unitoids. It is found that an $\mathscr{R}$-class of weakly inverse semigroup is a right skew groupoid $R=(R, P)$, where $P$ is a right skew semigroup [5], $P \subseteq R$, and $R$ is a partial semigroup satisfying certain conditions. When $S$ is a bisimple weakly inverse semigroup with $E$ the set of partial right unitoids, it can be shown that the $\mathscr{R}$-class $R=(R, P)$ containing $E$, which is a right skew groupoid, satisfies the following:

(i) for any $a, b \in R$, there exists $c \in R$ such that $P a \cap P b=P c$;

(ii) for any $a \in R$, there exists a left identity $e$ of $R$ such that $(P a \cap P) e=P a \cap P$.

Conversely, given a right skew groupoid $R$ satisfying the above conditions it is possible to construct a bisimple weakly inverse semigroup with partial right unitoids, having $R$ as an $R$-class. We recall [5] that an inverse semigroup with a system of partial identities is a monoid. On the other hand every inverse semigroup has a set of partial right unitoids, namely any singleton set $\{e\}$ with $e^{2}=e$. Thus the main results of [5] correspond to those of Clifford [3] whereas those of the present paper correspond to the results of Reilly [6].

2. Basic concepts. We assume that the reader is familiar with the basic results of [2 and 3].

Let $S$ be a semigroup. An idempotent $e$ of $S$ is called a principal idempotent of $S$ if $f e f=f e$ for every idempotent $f$ of $S$. An element $a$ of $S$ is called a principal element of $S$ if there exists an inverse $a^{\prime}$ of $a$ in $S$ such that $a a^{\prime}$ is a principal idempotent of $S$. It can be shown [7] that these two definitions are consistent. If $a$ is any element of $S$, then the inverse $a^{\prime}$ of $a$ will be called a principal inverse of $a$ if $a^{\prime} a$ is a principal idempotent of $S$. If $a \in S$, then $E_{a}$ will denote the set of principal inverses of $a$. Following [1 and 7] a semigroup $S$ is called a weakly inverse semigroup if for every $a \in S, E_{a} \neq \square$, and for every $a, b \in S$, we have

(i) $E_{a b} \subseteq E_{b} E_{a}$ and

(ii) $E_{a}=E_{b}$ implies $a=b$.

The following Lemma summarizes some of the results of [7].

LeMMA 2.1. Let $S$ be a weakly inverse semigroup. Then

(i) the principal idempotents of $S$ form a semilattice,

(ii) $E_{a} a$ consists of a single idempotent $e_{a}$ for every $a \in S$,

(iii) every principal left ideal of $S$ has a unique principal idempotent generator,

(iv) the set I of principal elements of $S$ forms an inverse subsemigroup of $S$,

Glasgow Math. J. 30 (1988) 1-10. 
(v) an element $a \in S$ is a principal element of $S$ if and only if a has a unique principal inverse,

(vi) for every $a, b \in S$ we have $E_{a b}=E_{b}^{a} E_{a}$ where $E_{b}^{a}=\left\{b^{\prime} \in E_{b}: e_{a} b b^{\prime} e_{a}=e_{a} b b^{\prime}\right\}$.

If $a$ is any element of the weakly inverse semigroup $S$, then $a^{\prime}, a_{1}^{\prime}, a_{2}^{\prime} \ldots$ will denote principal inverses of $a$ and $a^{\prime \prime}$ will denote the unique principal inverse of $a^{\prime} \in E_{a}$.

LEMMA 2.2. Let a be an element of a weakly inverse semigroup.

(i) If a Re for some idempotent $e$, then there is a principal inverse $a^{\prime}$ of a such that $a a^{\prime}=e$.

(ii) If $a^{\prime} \in E_{a}$ and $a^{\prime \prime} \in E_{a^{\prime}}$, then $a a^{\prime} a^{\prime \prime}=a$ and $a^{\prime \prime} a^{\prime} a=a^{\prime \prime}$.

Proof. (i) By Lemma 2.1.(iii), there is a unique principal idempotent $h$ in $L_{a}$. By [4, Proposition II.3.6], there is an inverse $a^{\prime}$ of $a$ in $R_{h} \cap L_{e}$ such that $a^{\prime} a=h$ and $a a^{\prime}=e$.

(ii) Since $a^{\prime}$ is principal, $a^{\prime} a^{\prime \prime}, a^{\prime} a$ are principal $\mathscr{R}$-related idempotents. Consequently, $a^{\prime} a^{\prime \prime}=a^{\prime} a$ and the result follows.

The semigroup $T(X)$ of partial transformations of the set $X$ is a weakly inverse semigroup. An element $\alpha \in T(X)$ is a principal element of $T(X)$ if and only if it is a one-to-one partial transformation of the set $X$. The semigroup $T(X)$ will be called the symmetric weakly inverse semigroup on the set $X$. We recall the following results [7].

LemMa 2.3. Let $S$ be a weakly inverse semigroup. For any $a \in S$, let $\psi_{a}$ be the partial transformation of $S$ where dom $\psi_{a}=S E_{a}$, and where for any $x \in \operatorname{dom} \psi_{a}, x \psi_{a}=x a$. The mapping $S \rightarrow T(S), a \rightarrow \psi_{a}$ embeds $S$ isomorphically into the symmetric weakly inverse semigroup $T(S)$ in such a way that an element $a \in S$ is principal in $S$ if and only if $\psi_{a}$ is principal in $T(S)$.

With the notation in the above lemma, we have the following.

LEMMA 2.4. Let $S$ be a weakly inverse semigroup, and let $a$ and $b$ be elements of $S$. The following conditions are equivalent:

(i) $E_{a} b=\left\{e_{a}\right\}$;

(ii) for every $a^{\prime} \in E_{a}$, there exists $b^{\prime} \in E_{b}$ such that $a^{\prime} \leqslant b^{\prime}$ in $I$;

(iii) $\psi_{a} \subseteq \psi_{b}$.

From Lemma 2.2 and Lemma 2.3, it follows that the relation $\leqslant$ on the weakly inverse semigroup $S$ which is defined by $a \leqslant b$ if $a$ and $b$ satisfy the equivalent conditions of Lemma 2.3 must be a partial order on $S$ which is compatible with multiplication. We shall call this partial order the natural partial order on the weakly inverse semigroup $S$. The natural partial order induces the usual partial order on the inverse subsemigroup of $S$. But $\leqslant$ does not induce the usual partial order on the idempotents of $S$ in the general case.

We cite the following results [5].

Leмma 2.5. If $S$ is any weakly inverse semigroup, then I is an order ideal of $S, \leqslant$.

LEMMA 2.6. If $e$ is a principal idempotent of the weakly inverse semigroup $S$ and $a \in S$, then $e a \leqslant a$ and $a e \leqslant a$. 
LEMMA 2.7. Let $S$ be a weakly inverse subsemigroup of the symmetric weakly inverse semigroup $T(S)$ on the set $S$. Let us suppose that for $\alpha \in S$ and for every $x \in \operatorname{dom} \alpha$ there exists a principal inverse $\alpha^{\prime}$ of $\alpha$ such that $x \alpha \alpha^{\prime}=x$. Then the natural partial order on $S$ coincides with the inclusion relation for partial transformations.

We add below an alternative characterization of weakly inverse semigroups, which will be used in the paper.

THEOREM 2.1. For a regular semigroup $S$, the following conditions are equivalent:

(i) $S$ is a weakly inverse semigroup;

(ii) there exists a commutative subsemigroup $C$ of idempotents such that

(a) for every $a \in S$, the set $C_{a}$ of inverses $a^{\prime}$ of a for which $a^{\prime} a \in C$ is non-empty,

(b) $C_{a b} \subseteq C_{b} C_{a}$ for all $a, b \in S$,

(c) $C_{a}=C_{b}$ implies $a=b$ for $a, b \in S$.

LeMma 2.8. Let $S$ be a weakly inverse semigroup and let $a \in S$. Let $a^{\prime}, a_{1}^{\prime} \in E_{a}$ be such that $a a^{\prime} \leqslant a a_{1}^{\prime}$. Then $a^{\prime}=a_{1}^{\prime}$.

Proof. Let $a a^{\prime} \leqslant a a_{1}^{\prime}$. Then $a^{\prime}=a^{\prime} a a^{\prime} \leqslant a^{\prime} a a_{1}^{\prime}=a_{1}^{\prime}$. Therefore, $a_{1}^{\prime} a_{1}^{\prime \prime}=a^{\prime} a^{\prime \prime}=a_{1}^{\prime} a^{\prime \prime}$. This implies $a_{1}^{\prime} \leqslant a^{\prime}$, whence we get $a^{\prime}=a_{1}^{\prime}$.

3. Right skew groupoids. We shall define a right partial binary operation on a set $S$ satisfying the following condition:

(A) if for elements $a, b, c$ of $S, a(b c)$ is defined, then also $(a b) c$ is defined and $a(b c)=(a b) c$.

Then $S$ is called a right partial semigroup.

A right skew groupoid $R=(R, P)$ is defined to be a right partial semigroup $R$ together with a subsemigroup $P$ of $R$ such that

$\mathrm{P}(1) a b$ is defined if and only if $a \in P$ for all $a, b \in R$,

$\mathrm{P}(2) a c=b c$ implies $a=b e$ where $e$ is a left identity of $R$, for $a, b \in P$ and all $c \in R$.

Remark. It can be verified that $P$ is a right skew semigroup [5]. The set of idempotents of $R$ coincides with the set of left identities of $R$ and they form a right zero semigroup. For any $a \in P$, there exists a left identity $e$ such that $a e=a$. Let $X$ be a set and $Y \subseteq X$. Let $\mu$ be an equivalence relation on $Y$. Let $T_{\mu}(X)$ be the set of partial transformations of $X$ with domain $Y$ such that

(i) $\operatorname{ker} \alpha=\mu$

(ii) $(x \alpha, y \alpha) \in \mu$ implies $(x, y) \in \mu$ for all $x, y \in X$.

On $T_{\mu}(X)$, a partial binary operation is defined thus: for $\alpha, \beta \in T_{\mu}(X), \alpha \beta$ is defined to be the usual composition of partial transformations if and only if $\operatorname{dom}(\alpha \beta)=Y$.

Since $\operatorname{dom}(\alpha \beta)=\left((\operatorname{ran}(\alpha) \cap \operatorname{dom}(\beta)) \alpha^{-1}\right.$ and $\operatorname{dom}(\beta)=Y$, we see that $\alpha \beta$ is defined if and only if $\operatorname{ran}(\alpha) \subseteq Y$. It can now be seen that if $\alpha \beta$ is defined, then $\alpha \beta \in T_{\mu}(X)$. For, if $x, y \in X$ and $(x, y) \in \mu$, then $x \alpha=y \alpha$ since $\alpha \in T_{\mu}(X)$. Conversely, if $x \alpha \beta=y \alpha \beta$, then $(x \alpha, y \alpha) \in \mu$ because ker $\alpha \beta=\mu$ and since $\alpha \in T_{\mu}(X)$, we get $(x, y) \in \mu$. Thus $\operatorname{ker}(\alpha \beta)=\mu$. A similar argument shows that if $(x \alpha \beta, y \alpha \beta) \in \mu$ then $(x, y) \in \mu$. 
We now define

$$
\bar{T}_{\mu}(X)=\left\{\alpha \in T_{\mu}(X): \operatorname{ran}(\alpha) \subseteq Y\right\} .
$$

THEOREM 3.1. $\left(T_{\mu}(X), \bar{T}_{\mu}(X)\right)$ is a right skew groupoid and every right skew groupoid $R=(R, P)$ can be faithfully represnted by partial transformations with domain $P$.

Proof. Let $\alpha, \beta, \gamma \in T(X)$ and let $\alpha(\beta \gamma)$ be defined. Then $\operatorname{ran}(\alpha) \subseteq Y$ and $\operatorname{ran}(\beta) \subseteq$ $Y$. Therefore, $\alpha \beta$ is defined. $\operatorname{ran}(\alpha \beta)=(\operatorname{ran}(\alpha) \cap \operatorname{dom}(\beta)) \beta \subseteq Y$. Thus $(\alpha \beta) \gamma$ is defined. Since the operation is the usual composition of binary relations it follows that $\alpha(\beta \gamma)=(\alpha \beta) \gamma$. It can be now verified that $\bar{T}_{\mu}(X)=\left\{\alpha \in T_{\mu}(X): \operatorname{ran}(\alpha) \subseteq Y\right\}$ is a right skew semigroup. Let now $\varphi \alpha=\psi \alpha$ for $\varphi, \psi \in T_{\mu}(X)$ and $\alpha \in T_{\mu}(X)$. Since $Y \psi$ intersects every $\mu$-class in at most one element, we can choose an idempotent $\varepsilon \in T_{\mu}(X)$ such that $Y \psi \subseteq Y \varepsilon$. As shown in [5], $\varepsilon \subseteq \mu$. Since $\operatorname{ker} \varepsilon=\operatorname{ker} \alpha=\mu$ it follows that $\varepsilon$ and $\alpha$ are $\mathscr{R}$-related in the semigroup of partial transformations of $X$. Therefore, $\varphi \alpha=\psi \alpha$ implies $\varphi \varepsilon=\psi \varepsilon$ where $\varphi \varepsilon=\psi$ since $Y \psi \subseteq Y \varepsilon$. We conclude that $T_{\mu}(X)$ is a right skew groupoid.

Conversely, when $R=(R, P)$ is a right skew groupoid, then

$$
\begin{aligned}
\mu & =\{(x, y) \in P \times P: x a=y a \text { for some } a \in R\} \\
& =\{(x, y) \in P \times P: x a=y a \text { for every } a \in R\}
\end{aligned}
$$

is a congruence on $R$. Define, for every $a \in R, \rho_{a}: P \rightarrow R$ by $x \rho_{a}=x a$. It is easily verified that $a \rightarrow \rho_{a}$ defines an isomorphism.

The proof of the theorem is now complete.

We shall now characterise an $\mathscr{R}$-class of a weakly inverse semigroup.

LEMma 3.1. Let $R$ be an $\mathscr{R}$-class of a weakly inverse semigroup. Then $R$ is a right skew groupoid.

Proof. For $a, b \in R$, we define $a \circ b=a b$ if and only if $a b \in R$. Define

$$
P=\{a \in R \text { : there exists a left identity } e \text { of } R \text { such that } a e=a\} .
$$

Let $a \circ b$ be defined for $a, b \in R$. Then $a b \in R$. Therefore there exist $a^{\prime} \in E_{a}$ and $b^{\prime} \in E_{b}^{a}$ such that $a b b^{\prime} a^{\prime}=a a_{1}^{\prime}$ for some $a_{1}^{\prime} \in E_{a}$. This implies $a b b^{\prime} a^{\prime} a=a$ and since $b^{\prime} \in E_{b}^{a}$ it follows that $a b b^{\prime}=a$. Since $b b^{\prime}$ is a left identity of $R$, we get $a \in P$. Conversely if $a \in P$ and $b \in R$, then there exists $b^{\prime} \in E_{b}$ such that $a b b^{\prime}=a$. Therefore $a b b^{\prime} a^{\prime}=a a^{\prime}$ for every $a^{\prime} \in E_{a}$ and consequently $a b \in R$. Now it can easily be verified that $R$ is a right partial semigroup. Let now $x a=y a$ for $x, y \in P$ and $a \in R$. Then there exists $a^{\prime} \in E_{a}$ such that $x a a^{\prime}=y a a^{\prime}$. Since $x \in P$, we can choose $a^{\prime} \in E_{a}$ such that $x a a^{\prime}=x$, and noting that $a a^{\prime}$ is a left identity of $R, \mathrm{P}(2)$ follows. The proof of the Lemma is now complete.

A right zero subsemigroup $E$ of a weakly inverse semigroup $S$ is called a system of partial right unitoids if for any $a \in S$ there exists $e \in E$ such that $a e \leqslant a$.

LEMMA 3.2. Let $S$ be a weakly inverse semigroup with a system of partial right unitoids 
$E$. Then the $R$-class $R=(R, P)$ which contains $E$ is a right skew groupoid satisfying the following:

$\mathrm{P}(3)$ for all $a \in R$, there exists $e \in E$ such that $(P a \cap P) e=P a \cap P$.

Proof. Let $a \in R$ and $e \in E$ such that $a e \leqslant a$. Then for any $x \in P a \cap P$, we have $x=y a$ for some $y \in P$ so that $x e=y a e \leqslant y a=x$.

Now there exists $(x e)^{\prime} \in E_{x e}$ such that $(x e)(x e)^{\prime}=e$, by Lemma 2.2. We have $(x e)^{\prime} \mathscr{L} e$ so that $(x e)^{\prime} x \mathscr{L} e x$. Now $e x=x$ and by Lemma 2.4. (i), $(x e)^{\prime} x=(x e)^{\prime} x e$ so that $x \mathscr{L} x e$.

Now $x=x f$ for some $f^{2}=f \in R$ since $x \in P$; since $x \mathscr{L} x e$, we have $x e=(x e) f=x(e f)=$ $x f=x$. The Lemma now follows.

In the remainder of the section, $R=(R, P)$ will denote a right skew groupoid and $E$ the set of idempotents of $R$. It is also assumed that the condition $\mathrm{P}(3)$ holds; i.e. for $a \in R$, there exists a left identity $e$ of $R$ such that $(P a \cap P) e=P a \cap P$. From Theorem 3.1, we know that there is a faithful representation $\rho$ of $R$ which maps $R$ isomorphically into the symmetric weakly inverse semigroup $T(R)$ of all partial transformations of $R$. For any $\alpha \in T(R)$, let $E_{\alpha}$ denote the set of principal inverses in $T(R)$. Define

$$
(R \rho)^{\prime}=\left\{\alpha^{\prime} \in E_{\alpha}: \alpha \in R \rho \text { and } \alpha \alpha^{\prime} \in R \rho\right\}
$$

and let $(R \rho)^{\prime \prime}=\left\{\alpha^{\prime \prime} \in E_{\alpha^{\prime}}: \alpha^{\prime} \in(R \rho)^{\prime}\right\}$.

Let $\Sigma$ be the subsemigroup of $T(R)$ which is generated by the elements of $R \rho \cup(R \rho)^{\prime} \cup(R \rho)^{\prime \prime}$. We shall show that the semigroup is a weakly inverse semigroup with a system of partial right unitoids.

LEMMA 3.3. For every $\alpha \in R \rho$ and every $\varepsilon=\varepsilon^{2} \in R$, there exists an $\alpha^{\prime} \in E_{\alpha} \cap(R \rho)^{\prime}$ such that $\alpha \alpha^{\prime}=\varepsilon$. $R \rho$ is an $R$-class of $\Sigma$.

Proof. Let $\alpha \in R \rho$ and $\varepsilon=\varepsilon^{2} \in R \rho$. Then $\alpha=a \rho$ and $\varepsilon=e \rho$ for some $a, e=e^{2} \in R$. The mapping $\alpha^{\prime}: P a \rightarrow P e, x a \rightarrow x e$ is a well-defined one-to-one partial transformation of the set $R$, and it is easy to see that $\alpha^{\prime} \in E_{\alpha} \cap(R \rho)^{\prime}$ and $\alpha \alpha^{\prime}=\varepsilon$. This incidentally shows that $R \rho$ is contained in an $R$-class of $\Sigma$.

If $\alpha \in R \rho$, then $\operatorname{dom}(\alpha)=P$ and $\alpha$ is a right translation of $P$. Let $x$ be any element of $R \rho$ and let $\alpha^{\prime} \in E_{\alpha}$, where $\alpha \alpha^{\prime} \in R \rho$. Let $s \in \operatorname{dom}(\alpha)$ and $s \alpha \alpha^{\prime}=q$. Since $\alpha^{\prime} \alpha$ is a restriction of the identity mapping to $\operatorname{dom}\left(\alpha^{\prime}\right)$, we have $s \alpha^{\prime} \alpha=q \alpha=s$. For any $r \in P$, $(r q) \alpha=r(q \alpha)=r s$ and so $r s \in \operatorname{dom}\left(\alpha^{\prime}\right)$. Also, $(r s) \alpha^{\prime}=(r q) \alpha \alpha^{\prime}=r\left(q \alpha \alpha^{\prime}\right)=r\left(s \alpha^{\prime}\right)$. Thus we can conclude that, whenever $s \in \operatorname{dom}\left(\alpha^{\prime}\right)$, then $(r s) \alpha^{\prime}=r\left(s \alpha^{\prime}\right)$, for all $r \in P$. In other words, $\alpha^{\prime}$ is a partial right translation for all $\alpha^{\prime} \in(R \rho)^{\prime}$. Let $\alpha^{\prime \prime} \in(R \rho)^{\prime \prime}$. Then $\alpha^{\prime \prime}=\alpha^{\prime \prime} \alpha^{\prime} \alpha$ and $\alpha^{\prime \prime} \alpha^{\prime} \in E_{\alpha \alpha^{\prime}} \cap(R \rho)^{\prime}$, and therefore $\alpha^{\prime \prime}$ is a partial right translation of $P$. Thus every element of $R \rho \cup(R \rho)^{\prime} \cup(R \rho)^{\prime \prime}$ is a partial right translation of $P$, and consequently all elements of $\Sigma$ are partial right translations. Let now $\xi$ be any element of the $\mathscr{R}$-class which contains $R \rho$ as a subset. Then $\operatorname{dom} \xi=P$ and so $\xi$ must be a right translation of $P$. If $f$ is any fixed left identity of $R$, then $f \rho$ is an idempotent of $R \rho$ and there exists $\xi^{\prime}$ in $\Sigma$ such that $\xi \xi^{\prime}=f \rho$. If $g$ is any left identity of $R$, then $g \xi \xi^{\prime} \xi=g(f \xi)=f \xi$. If $r$ is any element of $P$, then there exists a left identity $e$ of $R$ such that $r e=r$, and then $r \xi=(r e) \xi=r(e \xi)=r(f \xi)$. 
We conclude that $\xi=(f \xi) \rho \in R \rho$. Thus $R$ is an $\mathscr{R}$-class of $\Sigma$.

LEMMA 3.4. If $\alpha \in R \rho$, and $\beta^{\prime} \in(R \rho)^{\prime}$, then $\beta^{\prime} \alpha=\beta^{\prime} \alpha^{\prime \prime}$ where $\alpha^{\prime \prime} \in(R \rho)^{\prime \prime} \cap E_{\alpha^{\prime}}$ for some $\alpha^{\prime} \in(R \rho)^{\prime} \cap E_{\alpha}$ for which $\alpha \alpha^{\prime} \in R \rho$. If $\beta^{\prime \prime} \in(R \rho)^{\prime \prime}$, then $\beta^{\prime \prime} \alpha=\beta^{\prime \prime} \alpha_{1}^{\prime \prime}$ where $\alpha_{1}^{\prime \prime} \in$ $(R \rho)^{\prime \prime} \cap E_{\alpha_{1}}$ for some $\alpha_{1}^{\prime} \in(R \rho) \cap E_{\alpha}$ for which $\alpha \alpha_{1}^{\prime} \in R \rho$.

Proof. Since $\alpha \mathscr{R} \beta$, there exists $\alpha^{\prime} \in E_{\alpha} \cap(R \rho)^{\prime}$ such that $\alpha \alpha^{\prime}=\beta \beta^{\prime}$. Therefore, in view of Lemma 2.2(ii), $\beta^{\prime} \alpha=\beta^{\prime} \alpha \alpha^{\prime} \alpha=\beta^{\prime} \alpha \alpha^{\prime} \alpha^{\prime \prime}=\beta^{\prime} \beta \beta^{\prime} \alpha^{\prime \prime}=\beta^{\prime} \alpha^{\prime \prime}$. This proves the first part. To prove the second part, we note that there exist $b \in R$ and $e$ a left identity in $R$ such that $b \rho=\beta$ and $(P b \cap P) e=P b \cap P$. Consequently,

$$
\beta(e \rho) \subseteq \beta
$$

and

$$
\beta^{\prime \prime}(e \rho)=\beta^{\prime \prime} \beta^{\prime} \beta(e \rho) \subseteq \beta^{\prime \prime} .
$$

Also by Lemma 3.3, there exists $\alpha_{1}^{\prime} \in E_{\alpha} \cap(R \rho)^{\prime}$ such that $\alpha \alpha_{1}^{\prime}=e \rho$. We get

$$
\begin{aligned}
\beta^{\prime \prime} \alpha_{1}^{\prime \prime} & =\beta^{\prime \prime} \alpha_{1}^{\prime \prime} \alpha^{\prime} \alpha_{1}^{\prime \prime} \\
& =\beta^{\prime \prime} \alpha^{\prime \prime} \alpha_{1}^{\prime \prime} \alpha \text { by Lemma } 2.2, \\
& \subseteq \beta^{\prime \prime} \alpha \text { by Lemma } 2.7 .
\end{aligned}
$$

LEMMA 3.5. Let I be the subsemigroup of $\Sigma$ which is generated by the elements of $(R \rho)^{\prime} \cup(R \rho)^{\prime \prime}$. Then $I$ is an inverse subsemigroup of $\Sigma$, and all the elements of $I$ are principal in $\Sigma$. Moreover, $\Sigma=(R \rho) I \cup I$.

Proof. It is clear that $I$ consists of elements that are principal in $T(R)$. Therefore it is a subsemigroup of the symmetric inverse semigroup on $R$. Since $I$ is generated by a set of elements and their inverses, it is an inverse subsemigroup of the symmetric inverse semigroup on $R$. All the idempotents of $I$ are principal in $T(R)$, and therefore all the elements of $I$ are principal in $\Sigma$. To show the last part, let $\alpha, \beta \in R \rho$. Then there exists $\beta^{\prime} \in E_{\beta} \cap(R \rho)^{\prime}$ such that $\alpha \beta \beta^{\prime} \subseteq \alpha$ and therefore, $\alpha \beta=\alpha \beta \beta^{\prime} \beta^{\prime \prime} \subseteq \alpha \beta^{\prime}$. Thus $\alpha \beta=\alpha \beta^{\prime \prime}$. With the preceding lemma, it can now be observed that $\Sigma=(R \rho) I \cup I$.

Lemma 3.6. For any $\xi \in \Sigma$, let $G_{\xi}$ denote the set of inverses $\xi^{\prime}$ of $\xi$ in $\Sigma$ such that $\xi \xi^{\prime} \in I$. Then $G_{\xi}=E_{\xi} \cap \Sigma \neq \square$. For every $\alpha \in R \rho$ and every $\zeta \in I$, we have $G_{\alpha \zeta}=G_{\xi} G_{\alpha}$.

Proof. If $\xi \in \Sigma$, then $\xi \in I$ or $\xi \in(R \rho) I$. If $\xi \in I$, then $G_{\xi}=E_{\xi}=E_{\xi} \cap \Sigma$. Let $\xi \in(R \rho) I$. Then $\xi=\alpha \zeta$ where $\alpha \in R \rho$ and $\zeta \in I$. By Lemma 3.3, $G_{\alpha} \neq \square$. If $\zeta^{\prime}$ is the unique principal inverse of $\zeta$ in $I$ and $\alpha^{\prime} \in G_{\alpha} \subseteq E_{\alpha} \cap \Sigma$, then $\zeta^{\prime} \alpha^{\prime}$ is an element of $I$, which is an inverse of $\alpha \zeta$, where $\zeta^{\prime} \alpha^{\prime} \alpha \zeta$ is an idempotent of $I$. Consequently, $\square \neq G_{\zeta} G_{\alpha} \subseteq E_{\alpha \zeta} \cap \Sigma$. Let now $(\alpha \zeta)^{\prime}$ be an element of $E_{\alpha \zeta} \cap \Sigma$. Since $E_{\alpha \zeta} \subseteq E_{\zeta} E_{\alpha}=$ $\zeta^{\prime} E_{\alpha}$, where $\zeta^{\prime}$ is the unique principal inverse of $\zeta$ in $I$, we must have $(\alpha \zeta)^{\prime}$ in the form $\zeta^{\prime} \alpha_{1}^{\prime}$, where $\alpha_{1}^{\prime} \in E_{\alpha}$. We note that $(\alpha \zeta)\left(\zeta^{\prime} \alpha_{1}^{\prime}\right) \in \Sigma$. Then $\zeta^{\prime} \alpha_{1}^{\prime}=\beta_{1} \beta_{2} \ldots \beta_{n}$, where $\beta_{i} \in$ $R \rho \cup(R \rho)^{\prime} \cup(R \rho)^{\prime \prime}$ for all $i=1,2, \ldots, n$. Since $\zeta^{\prime} \alpha_{1}^{\prime} \in I$, we may suppose that $\beta_{n} \in$ $(R \rho)^{\prime}$ or $\beta_{n} \in(R \rho)^{\prime \prime}$. If $\beta_{n} \in(R \rho)^{\prime}$, there exists a left identity $\varepsilon$ of $R \rho$ such that $\beta_{n} \varepsilon=\beta_{n}$ and thus $\zeta^{\prime} \alpha_{1}^{\prime} \varepsilon=\zeta^{\prime} \alpha_{1}^{\prime}$. It can be verified that $\alpha^{\prime} \varepsilon \in G_{\alpha}$. If $\beta_{n} \in(R \rho)^{\prime \prime}$, there exists a left 
identity $\varepsilon$ of $R \rho$ such that $\beta_{n} \varepsilon=\beta_{n}$. Thus $\zeta^{\prime} \alpha_{1}^{\prime} \leqslant \zeta^{\prime} \alpha^{\prime}$ for some $\alpha^{\prime} \in G_{\alpha}$ and thus $\alpha \zeta \zeta^{\prime} \alpha_{1}^{\prime} \leqslant \alpha \zeta \zeta^{\prime} \alpha^{\prime}$. This implies, in view of Lemma 2.8 , that $\zeta^{\prime} \alpha_{1}^{\prime}=\zeta^{\prime} \alpha^{\prime}$.

Thus we have shown that $G_{\alpha \zeta} \subseteq G_{\zeta} G_{\alpha}$. This together with the earlier assertion proves the Lemma.

LEMMA 3.7. $\Sigma$ is weakly inverse.

Proof. Let $\xi, \eta \in \Sigma$. If $\xi, \eta \in I$, then it is clear that $G_{\xi \eta}=G_{\eta} G_{\xi}$. If $\xi, \eta \in(R \rho) I$, let $\xi=\alpha \zeta$ and $\eta=\beta \theta$ for $\alpha, \beta \in R \rho$ and $\zeta, \theta \in I$. By Lemma 3.4, there exists $\beta^{\prime \prime} \in I$ with $G_{\beta}^{\prime \prime} \subseteq G_{\beta}$ such that $\zeta \beta=\zeta \beta^{\prime \prime}$. We have,

$$
\begin{aligned}
G_{\alpha \zeta \beta \theta} & =G_{\alpha \zeta \beta^{\prime \prime} \theta} \\
& =G_{\zeta \beta^{\prime \prime} \theta} G_{\alpha} \\
& =G_{\beta^{\prime \prime} \theta} G_{\zeta} G_{\alpha} \\
& =G_{\beta^{\prime \prime} \theta} G_{\alpha \zeta} \\
& \subseteq G_{\beta \theta} G_{\alpha \zeta} \\
& =G_{\eta} G_{\xi} .
\end{aligned}
$$

The other cases may be dealt with in a similar fashion, whence it follows that $G_{\xi \eta} \subseteq G_{\eta} G_{\xi}$ for all $\xi, \eta \in \Sigma$.

Let $\xi=\alpha \zeta, \quad(\alpha \in R \rho, \zeta \in I)$ be any element of $(R \rho) I$ and let $G_{\xi}$ be singleton. If $x \alpha \zeta=y \alpha \zeta$ for $x, y \in P$, then $x \alpha=y \alpha$. Put $\alpha=a \rho$; then $x a=y a$ and since $R$ is a right skew groupoid, $x=y e$ for some left identity $e$ of $R$. If $\varepsilon=e \rho$, then there exists $\alpha^{\prime} \in G_{\alpha}$ such that $\alpha \alpha^{\prime}=\varepsilon$. If $\left\{\zeta^{\prime}\right\}=G_{\zeta}$, then $\zeta^{\prime} \alpha^{\prime} \in G_{\alpha \zeta}$. If $u=y \alpha \zeta \zeta^{\prime} \alpha^{\prime}$, then $u \alpha \zeta \zeta^{\prime} \alpha^{\prime}=$ $y \alpha \zeta \zeta^{\prime} \alpha^{\prime}$ and hence $u \alpha \zeta=y \alpha \zeta$. Again $y=u \lambda$ for some left identity $\lambda$ of $R \rho$, and there exists $\alpha_{1}^{\prime} \in G_{\alpha}$ such that $\alpha \alpha_{1}^{\prime}=\lambda$. Since both $\zeta^{\prime} \alpha^{\prime}$ and $\zeta^{\prime} \alpha_{1}^{\prime}$ belong to $G_{\alpha \zeta}$ and since $G_{\alpha \zeta}$ is singleton, we have $\zeta^{\prime} \alpha^{\prime}=\zeta^{\prime} \alpha_{1}^{\prime}$. Therefore,

$$
y=u \lambda=y \alpha \zeta \zeta^{\prime} \alpha^{\prime} \lambda=y \alpha \zeta \zeta^{\prime} \alpha_{1}^{\prime} \alpha \alpha_{1}^{\prime}=y \alpha \zeta \zeta^{\prime} \alpha_{1}^{\prime}=y \alpha \zeta \zeta^{\prime} \alpha^{\prime}=u
$$

and so

$$
u=u \alpha \alpha^{\prime}=u e=y e=x .
$$

Thus $\xi=\alpha \zeta$ is a one-to-one partial transformation on $R$, and $\xi$ is a principal element of $T(R)$.

If $\xi$ and $\eta$ are any elements of $\Sigma$ such that $G_{\xi}=G_{\eta}$, and if $\eta \in I$, then $G_{\xi}=G_{\eta}=E_{\eta}$ is singleton. By the earlier assertion, it follows that $\xi$ must be principal in $T(R)$, hence $G_{\xi}=E_{\xi}$. Now $E_{\xi}=E_{\eta}$ implies $\xi=\eta$.

Let us now suppose that $\xi=\alpha \zeta$ and $\eta=\beta \theta$ where $\alpha, \beta \in R \rho$ and $\zeta, \theta \in I$ and $G_{\xi}=G_{\eta}$. Every element of $G_{\xi}$ is of the form $\zeta^{\prime} \alpha^{\prime}=\xi^{\prime}$ with $\zeta^{\prime} \in G_{\zeta}$ and $\alpha^{\prime} \in G_{\alpha}$. Then $\xi^{\prime} \in G_{\xi}$ and so $\xi^{\prime} \eta=\xi^{\prime} \xi$. Since $\alpha \alpha^{\prime}$ is a left identity for $R \rho$ we have $\alpha \alpha^{\prime} \eta=\alpha \alpha^{\prime}=\beta \theta=$ $\eta$. Since $\zeta^{\prime} \zeta$ is a restriction of the identity transformation to $\operatorname{dom}\left(\zeta^{\prime} \zeta\right)$ we have $\xi \zeta^{\prime}=\alpha \zeta \zeta^{\prime} \alpha \subseteq \alpha \alpha^{\prime}$. Therefore $\xi=\xi \xi^{\prime} \xi=\xi \xi^{\prime} \eta \subseteq \alpha \alpha^{\prime} \eta=\eta$. The reverse inclusion can similarly be proved, whence it follows that $\xi=\eta$. 
The Lemma now follows.

Lemma 3.8. The set of idempotents of the $\mathscr{R}$-class $R \rho$ forms a system of partial right unitoids of $\Sigma$.

Proof. We first note that if $\alpha \in I$, then $\alpha=\alpha_{1} \alpha_{2} \ldots \alpha_{n}$ where $\alpha_{n} \in(R \rho)^{\prime} \cup(R \rho)^{\prime \prime}$. If $\alpha_{n} \in(R \rho)^{\prime}$, then there exists $\beta \in R \rho$ such that $\alpha_{n}=\beta^{\prime} \in G_{\beta}$. Then $\alpha_{n}\left(\beta \beta^{\prime}\right)=\alpha_{n}$. When $\alpha_{n} \in(R \rho)^{\prime \prime}$, there exists $\beta \in R \rho$ such that $\alpha_{n}=\beta^{\prime \prime} \in G_{\beta^{\prime}}$. As observed in the proof of Lemma 3.4, there exists a left identity $\varepsilon$ of $R \rho$ such that $\alpha_{n} \varepsilon \subseteq \alpha_{n}$. Combining the two we get

$$
\alpha \varepsilon=\alpha_{1} \alpha_{2} \ldots \alpha_{n} .
$$

Since $\Sigma=(R \rho) I \cup I$, the result follows.

The preceding lemmas combine to prove

THEOREM 3.2. Let $R=(R, P)$ be a right skew groupoid and $R \rho$ its representation defined earlier. Let $\Sigma=\left\langle R \rho \cup(R \rho)^{\prime} \cup(R \rho)^{\prime \prime}\right\rangle$, the semigroup generated by $R \rho \cup(R \rho)^{\prime} \cup$ $(R \rho)^{\prime \prime}$. Then $\Sigma$ is weakly inverse and it has a system of partial right unitoids contained in $R \rho$.

\section{Bisimple weakly inverse semigroups with partial right unitoids.}

THEOREM 4.1. Let $S$ be a bisimple weakly inverse semigroup with a system $E$ of partial right unitoids. Then the $\mathscr{R}$-class $R=(R, P)$ containing $E$ is a right skew groupoid satisfying the following conditions:

$P(3)$ for all $a \in R$, there exists $e \in E$ such that $(P a \cap P) e=P a \cap P$;

$P(4)$ for all $a, b \in R$, there exists $c \in R$ such that $P a \cap P b=P c$.

Proof. In view of Lemmas 3.1 and 3.2, it is enough to show that $\mathrm{P}(4)$ is satisfied. For this, let $a \in R$. To show the reverse inclusion, let $x \in S a \cap R$. Then there exists $s \in S$ such that $x=s a \in R$. Then there exists $a^{\prime} \in E_{a}$ and $s^{\prime} \in E_{s}$ such that $s a a^{\prime} s^{\prime} \in R$. Clearly, $s a a^{\prime} a^{\prime \prime} a^{\prime} s^{\prime} \in R$ and it follows that $s a a^{\prime} \in R$ and $a a^{\prime}$ is a left identity of $R$; we get $s a a^{\prime} \in P$. It now follows that $s a=s a a^{\prime} a \in P a$. Then

$$
\begin{aligned}
P a \cap P b & =S a \cap R \cap S b \cap R=S a \cap S b \cap R \\
& =S e_{a} \cap S e_{b} \cap R=S e_{a} e_{b} \cap R .
\end{aligned}
$$

Since $S$ is bisimple and $R$ is an $\mathscr{R}$-class, there exists $c$ such that $S e_{a} e_{b}=S c$. We thus get $\mathrm{P}(4)$. The proof of the Theorem is now complete.

THEOREM 4.2. Let $R=(R, P)$ be a right skew groupoid satisfying the following conditions:

$\mathrm{P}(3)$ for all $a \in R$, there exists a left identity e of $R$ such that $(P a \cap P) e=P a \cap P$;

$\mathrm{P}(4)$ for all $a, b \in R$, there exists $c \in R$ such that $P a \cap P b=P c$.

Then $\Sigma=\left\langle R \rho \cup(R \rho)^{\prime} \cup(R \rho)^{\prime \prime}\right\rangle$ is a bisimple weakly inverse semigroup with partial right unitoids. 
Proof. In view of Lemmas 3.7 and 3.8, it is enough to show that $\Sigma$ is bisimple. We first note that

$$
\Sigma=\left\langle R \rho \cup(R \rho)^{\prime} \cup(R \rho)^{\prime \prime}\right\rangle=\left\langle R \rho \cup(R \rho)^{\prime}\right\rangle .
$$

For this let $\alpha \in R \rho, \alpha^{\prime} \in(R \rho)^{\prime} \cap E_{\alpha}$ and $\alpha^{\prime \prime} \in(R \rho)^{\prime \prime} \cap E_{\alpha}$. Then $\alpha^{\prime \prime}=\alpha^{\prime \prime} \alpha^{\prime} \alpha$.

It can be checked that $\alpha^{\prime \prime} \alpha^{\prime} \in(R \rho)^{\prime} \cap E_{\alpha \alpha^{\prime}}$, where $\alpha \alpha^{\prime} \in R \rho$. Thus it follows that $\alpha^{\prime \prime} \in R \rho \cup(R \rho)^{\prime}$. Therefore

$$
\left\langle R \rho \cup(R \rho)^{\prime} \cup(R \rho)^{\prime \prime}\right\rangle \subseteq\left\langle R \rho \cup(R \rho)^{\prime}\right\rangle
$$

The reverse inclusion is obvious, whence it follows that

$$
\left\langle R \rho \cup\left(R(\rho)^{\prime}\right\rangle=\left\langle R \rho \cup(R \rho)^{\prime} \cup(R \rho)^{\prime \prime}\right\rangle .\right.
$$

To show that $\Sigma$ is bisimple, let $\alpha, \beta \in R \rho$. Let $\gamma$ be an element of $R \rho$ such that $(P \rho) \alpha \cap(P \rho) \beta=(P \rho) \gamma$. Putting $G_{\alpha} \alpha=\left\{e_{\alpha}\right\}$ and $G_{\beta} \beta=\left\{e_{\beta}\right\}$, it follows that $e_{\alpha} e_{\beta}=e_{\gamma}$, since $e_{\gamma}$ (resp. $\left.e_{\alpha}, e_{\beta}\right)$ is the identity mapping on $R_{\gamma}=R_{\alpha} \cap R_{\beta}\left(\operatorname{resp}, R_{\alpha}, R_{\beta}\right)$. If $\left(\alpha \beta^{\prime} \beta\right)^{\prime}$ is any element of $G_{\alpha \beta^{\prime} \beta}=\beta^{\prime} \beta G_{\alpha}$, then $\left(\alpha \beta^{\prime} \beta\right)^{\prime} \alpha \beta^{\prime} \beta=e_{\beta} e_{\alpha} e_{\beta}=e_{\gamma}$. Therefore, $\alpha \beta^{\prime} \mathscr{R} \alpha \beta^{\prime} \beta \mathscr{L} \gamma$, and so $\alpha \beta^{\prime}$ belongs to the $\mathscr{D}$-Class which contains $R \rho$ as an $\mathscr{R}$-Class. Let $\alpha^{\prime} \in(R \rho)^{\prime} \cap E_{\alpha}$ such that $\alpha \alpha^{\prime}=\beta \beta^{\prime}$, then $\beta^{\prime} \mathscr{L} \alpha^{\prime}$ and $\beta^{\prime} \alpha \mathscr{L} \alpha^{\prime} \alpha \mathscr{L} \alpha$ and so $\beta^{\prime} \alpha$ belongs to the $\mathscr{D}$-Class which contains $R \rho$. If $\alpha_{1}^{\prime}$ is any element of $(R \rho)^{\prime} \cap E_{\alpha}$, then $\alpha_{1}^{\prime} \beta^{\prime} \mathscr{L}\left(\alpha \alpha_{1}^{\prime}\right) \beta_{1}^{\prime}$ where $\alpha \alpha_{1}^{\prime} \in R \rho$, and by the foregoing we can conclude that $\alpha_{1}^{\prime} \beta^{\prime}$ belongs to the $\mathscr{D}$-Class that contains $R \rho$. Again, let $\alpha, \beta \in R \rho$. There exists $\beta^{\prime} \in E_{\beta} \cap(R \rho)^{\prime}$ such that $\alpha \beta \beta^{\prime} \subseteq \alpha$. Therefore, $\alpha \beta=\alpha \beta \beta^{\prime} \beta^{\prime \prime} \subseteq \alpha \beta^{\prime \prime} \subseteq \alpha \beta$ and we get $\alpha \beta=\alpha \beta^{\prime \prime}$. It is easy to observe that $\alpha \beta^{\prime \prime} \mathscr{L} \alpha^{\prime} \beta^{\prime \prime} \mathscr{L} \beta^{\prime} \alpha^{\prime} \alpha^{\prime \prime} \beta^{\prime \prime} \mathscr{R} \beta^{\prime} \alpha^{\prime}$. Consequently, $\alpha \beta=\alpha \beta^{\prime \prime}$ belongs to the $\mathscr{R}$-Class containing $R \rho$. Thus we have shown that the product of any two elements of $R \rho \cup(R \rho)^{\prime}$ belongs to the $\mathscr{D}$-Class which contains $R \rho$. Let $\xi$ be any element of this $\mathscr{D}$-Class and let $\zeta$ be any element of $R \rho \cup(R \rho)^{\prime}$. If $\gamma \in L_{\xi} \cap R \rho$, then $\gamma \zeta$ belongs to the $\mathscr{D}$-Class which contains $R \rho$. Since $\xi \zeta \mathscr{L} \gamma \zeta$ this implies that $\xi \zeta$ belongs to this $\mathscr{D}$-Class. By induction, it follows that $\Sigma$ is bisimple. $P(4)$.

ExAMPLE. We add below an example of a right skew groupoid satisfying $P(3)$ and

Let $R=\{\ldots-2,-1,0,1,2, \ldots\}$ be the group of integers under addition and let $P=\{0,1,2, \ldots\}$.

Let $R^{\prime}=\left\{\ldots-2^{\prime},-1^{\prime}, 0,1,2, \ldots\right\}$ be an isomorphic group with $R \cap R^{\prime}=P$. Put $S=R \cup R^{\prime}$ and define a partial operation $*$ by the rule $a * b=a+b$ if and only if $a \in P$. Then $(S, P)$ is a right skew groupoid satisfying $\mathrm{P}(3)$ and $\mathrm{P}(4)$. It is easy to verify that $(S, P)$ is a right skew groupoid having $P$ as its right skew subsemigroup. Since $S$ has an identity, $\mathrm{P}(3)$ holds. To show that $\mathrm{P}(4)$ holds we note that for any $a \in S$, we have

$P * a=\{x \in S: a \leqslant x\}$ where $\leqslant$ is the order relation in $R$ if $a \in R$ and the order relation in $R^{\prime}$ if $a \in R^{\prime}$. In all cases $((P * a) \cap P) * 0=(P * a) \cap P$, whence $\mathrm{P}(4)$ follows. 


\section{REFERENCES}

1. R. J. Brown, D. W. Hardy and R. J. Painter, A note on a paper by Srinivasan, Math. Ann. 186 (1970), 34-35.

2. A. H. Clifford and G. B. Preston, The algebraic theory of semigroups, Mathematical Surveys 7, vol. I (American Mathematical Society, 1961).

3. A. H. Clifford and G. B. Preston, The algebraic theory of semigroups, Mathematical Surveys 7, vol. II (American Mathematical Society, 1967).

4. J. M. Howie, An introduction to semigroup theory, London Mathematical Society Monographs 7 (Academic Press, 1976).

5. S. Madhavan, On bisimple weakly inverse semigroups, Pacific J. Math. 90 (1980), 397-409.

6. N. R. Reilly, Bisimple inverse semigroups, Trans. Amer. Math. Soc. 132 (1968), 101-114.

7. B. R. Srinivasan, Weakly inverse semigroups, Math. Ann. 176 (1968), 324-333.

Department of Mathematics

UNIVERSITY COLLEGE

TRIVANDRUM 695034

KERALA

SOUTH INDIA 